\title{
Review Article \\ Pancreatic Perfusion CT in Early Stage of Severe Acute Pancreatitis
}

\author{
Yoshihisa Tsuji, ${ }^{1,2}$ Naoki Takahashi, ${ }^{2}$ and Chiba Tsutomu ${ }^{1}$ \\ ${ }^{1}$ Department of Gastroenterology and Hepatology, Kyoto University Graduate School of Medicine, Shogoin, \\ Sakyo-ku, Kyoto 606-8507, Japan \\ ${ }^{2}$ Division of Radiology Mayo Clinic, Rochester MN 55905, USA \\ Correspondence should be addressed to Chiba Tsutomu, chiba@kuhp.kyoto-u.ac.jp \\ Received 16 September 2011; Revised 8 November 2011; Accepted 10 November 2011 \\ Academic Editor: Marja-Leena Kylanpaa
}

Copyright (๑) 2012 Yoshihisa Tsuji et al. This is an open access article distributed under the Creative Commons Attribution License, which permits unrestricted use, distribution, and reproduction in any medium, provided the original work is properly cited.

Early intensive care for severe acute pancreatitis is essential for improving SAP mortality rates. However, intensive therapies for SAP are often delayed because there is no ideal way to accurately evaluate severity in the early stages. Currently, perfusion CT has been shown useful to predict prognosis of SAP in the early stage. In this presented paper, we would like to review the clinical usefulness and limitations of perfusion CT for evaluation of local and systemic complications in early stage of SAP.

\section{Introduction}

Severe acute pancreatitis (SAP) is a fatal disease [1]. The Atlanta Symposium criteria for the severity of acute pancreatitis define SAP as acute pancreatitis with local complications (pancreatic necrosis, abscess, and pseudocysts) and/or with systemic complications (organ failure, disseminated intravascular coagulation, and severe metabolic disturbances) [2] (Figure 1). Both acute necrotizing pancreatitis (ANP) and multiple-organ failure (MOF) have been shown to be significant prognostic factors [3-6]. Mortality rates for SAP patients developing ANP and MOF are 32\% and 30\%, respectively [7]. Early intensive care for SAP is essential for improving SAP mortality rates [8-10]. However, intensive therapies for SAP are often delayed because there is no ideal way to accurately evaluate severity in the early stages [11-13].

Perfusion CT has been used for evaluation of various pancreatic diseases [14-22]. Perfusion CT has been shown useful to predict prognosis of SAP in the early stage $[17,18]$. In this presented paper, we would like to review the clinical usefulness and limitations of perfusion CT for evaluation of local and systemic complications in early stage of SAP.

\section{Perfusion CT Technique}

Previously published perfusion CT protocols are summarized in Table 1. Multidetector CT (MDCT) is essential for performing perfusion CT of pancreas. With a 4-64 slice MDCT scanner, perfusion CT scans are obtained with the patient in a stationary position. The craniocaudal CT scan coverage is limited to 20 to $32 \mathrm{~mm}$ (4 slices of 5 to $8 \mathrm{~mm}$ thickness). Therefore, scan location must be carefully chosen to cover as much of the pancreas as possible as it is often difficult to cover the entire pancreas. Since most pancreatic necrosis occurs in the neck region, it is probably uncommon to exclude the area of necrosis due to the scanning coverage limitation. With the use of a recently developed 256320-slice MDCT scanners [19], craniocaudal coverage has increased to $80-160 \mathrm{~mm}$. Alternative way to increase the craniocaudal coverage is by using the so-called shuttle or toggle mode. In this mode, similar to conventional CT scans, patient table moves back and forth as the multiple scans are performed.

First, noncontrast transaxial images of the upper abdomen are obtained using low-dose technique. This scan is performed to localize the pancreas, and it determines the scan range of the perfusion CT.

Perfusion CT is performed after a bolus injection of intravenous contrast material. Unlike conventional CT, the perfusion CT requires smaller dose $(40-50 \mathrm{~mL})$ of contrast material injected at a high rate $(4-10 \mathrm{~mL} / \mathrm{sec})$. Higher concentration of contrast material $(350-370 \mathrm{mgI} / \mathrm{kg})$ is preferred [23-25]. 
Perfusion CT images are obtained multiple times through the pancreas. In most of previous reports, scan interval ranges from 0.5 to 1.5 second, and the scan duration ranges from 30 to 150 seconds, respectively (Table 1). Total scan duration necessary for calculation of perfusion parameters may depend on the algorithm used. For example, maximum slope method needs shorter duration scan time than the deconvolution method $[14,19,26]$. Because the scan duration is long, the scans are usually performed under free breathing.

Perfusion CT scan is obtained at a low tube current (mAs) to reduce radiation dose. At $120 \mathrm{kV}$, mAs of 100 is commonly used. There is increased interest in the use of low tube voltage setting, as it reduces radiation dose and improves iodine contrast material conspicuity. In a smaller patient, the use of $100 \mathrm{kV}$ or $80 \mathrm{kV}$ is recommended. In a larger patient, the use of low-kV scan may result in noisy images due to photon deficiency.

2.1. Radiation Dose and Scan Parameters. Radiation dose is dependent on the tube current (mAs), tube voltage $(\mathrm{kV})$, number of scans, and scan coverage [29]. Tube current (mAs) and tube voltage $(\mathrm{kV})$ are largely dictated by the patient size to maintain adequate image quality. Radiation dose should be kept as low as reasonably achievable (ALARA) by reducing the scanning parameter settings but achieving image dataset adequate for calculating CT perfusion parameters [27, 30]. Further study is necessary to optimize the scanning protocol.

From a European study, the effective dose of pancreatic perfusion CT was $3.54 \mathrm{mSv}$ with $90 \mathrm{KV}, 100 \mathrm{mAs}$, and 40 scans [17]. A study from Japan reported that mean radiation dose of pancreatic perfusion CT was approximately $204.8 \mathrm{mGy} \cdot \mathrm{cm}$ (dose-length product (DLPw)), 3.07 $\mathrm{mSv}$ (effective dose), and $64 \mathrm{mGy}$ (CT dose index volume (CTDIvol)) with $80 \mathrm{kV}, 60 \mathrm{mAs}$, and 106 scans [27]. In the national survey, the radiation exposure of a single-phase abdominal CT was 13-25 mGy (CTDIvol) [31]. Therefore, the radiation dose of perfusion CT is slightly higher than that of biphasic (two phase), which is commonly used for pancreatic or liver imaging. Average abdominal transverse diameter of the Japanese patients in our experience was $32 \mathrm{~cm}$, while transverse diameters of patients in the Unites States are usually larger [32]. Therefore, the radiation dose will likely be higher in the western countries

2.2. Perfusion CT Data Analysis. Pancreatic perfusion CT image data are analyzed by using perfusion CT analysis software. There are various perfusion algorithms to calculate perfusion parameters. Maximum slope method, deconvolution method, single-compartment method, and the Patlak method are commonly used perfusion algorithms. Which perfusion best suits in the evaluation of SAP is yet to be determined. As different perfusion algorithms are suited for different disease processes and require different scanning protocol, determination of scanning protocol and perfusion algorithms should be considered together. For example, maximum slope method may require shorter scanning duration, but higher rate of contrast injection is required,

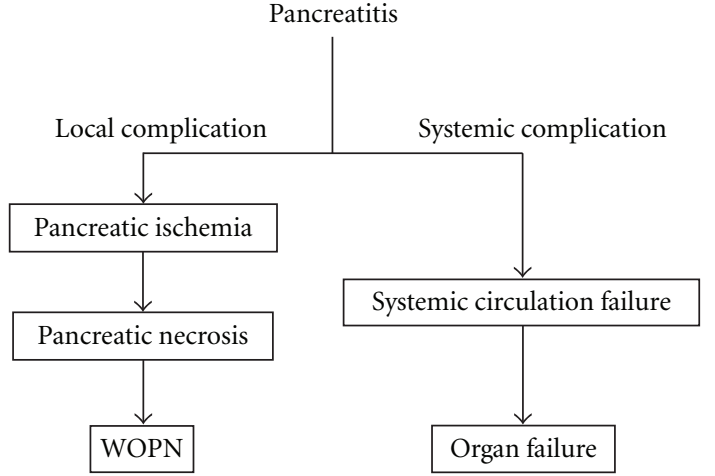

FIGURE 1: Schema of complications of severe acute pancreatitis. WOPN: walled-off pancreatic necrosis.

while deconvolution method may require longer scanning duration but slower rate of contrast injection rate $[14,26]$.

The software requires placement of small regions of interest (ROI) on an artery to generate arterial input function. Venous outflow function is required in deconvolution method. This process is required because the computer algorithm compares the shape and height of the timedensity curve of each pixel of the pancreatic CT time series with shape and height of the arterial and/or venous timedensity curves to calculate pancreatic perfusion parameters. Calculated pancreatic perfusion parameters are displayed using color maps [14].

\section{Perfusion CT for Predicting Development of Pancreatic Necrosis in the Early Stage of Severe Acute Pancreatitis}

Development of pancreatic necrosis is the critical event of acute pancreatitis that determines the prognosis of the patients. The overall mortality rate of acute pancreatitis is reported to be between $2.1 \%$ and $9.2 \%$ worldwide [1]. Pancreatic necrosis occurs in $10-15 \%$ of patients with SAP, with a mortality rate of $23 \%$ [1]. This rate is nearly twice that for patients with SAP who do not develop pancreatic necrosis (i.e., $11 \%$ ) [1].

There is a report that dynamic contrast-enhanced CT is more accurate than either the Ranson criteria for pancreatitis mortality or the APACHE II scoring system in predicting the development of pancreatic necrosis [33]. However, the accuracy of contrast-enhanced CT in predicting necrosis at an early stage of SAP is not satisfactory [34]. The United Kingdom guidelines for the management of acute pancreatitis, the most popular clinical guideline of acute pancreatitis, recommends that contrast-enhanced CT should be performed at day 3 or later after onset of SAP because of its low sensitivity of CT [11].

In our experience, perfusion CT performed within 3 days of onset of symptoms had a sensitivity and specificity of $100 \%$ and $95.3 \%$ for predicting development of pancreatic necrosis [18]. The area of necrosis was depicted as area 
TABLE 1: Scanning protocols of pancreatic perfusion CT.

\begin{tabular}{|c|c|c|c|c|c|c|c|c|c|c|}
\hline \multirow[t]{2}{*}{ Authors } & \multirow[t]{2}{*}{ Disease } & \multirow[t]{2}{*}{ CT } & \multirow{2}{*}{$\begin{array}{c}\text { The } \\
\text { number of } \\
\text { detector }\end{array}$} & \multirow[t]{2}{*}{$\mathrm{kv}$} & \multirow[t]{2}{*}{$\mathrm{mA}$} & \multirow[t]{2}{*}{ Images } & \multicolumn{2}{|c|}{ Contrast matter } & \multirow{2}{*}{$\begin{array}{l}\text { Duration } \\
\text { time }(\mathrm{sec})\end{array}$} & \multirow[t]{2}{*}{ Algorithm } \\
\hline & & & & & & & $\begin{array}{l}\text { Injection rate } \\
(\mathrm{mL} / \mathrm{sec})\end{array}$ & Amount & & \\
\hline \multirow{3}{*}{ Miles [25] } & - & - & - & - & $50-100$ & 60 & $4-7$ & $40 \mathrm{~mL}$ & 60 & Deconvolution \\
\hline & & & & & $100-250$ & 15 & $7-10$ & $50 \mathrm{~mL}$ & 45 & Compartment \\
\hline & & & & & $100-250$ & 6 & 4 & $100 \mathrm{~mL}$ & 120 & Patlak plot \\
\hline $\begin{array}{l}\text { Tsushima and } \\
\text { kusano [15] }\end{array}$ & Normal & S & 1 & - & - & 19 & 5 & $40 \mathrm{~mL}$ & 85 & Maximum slope \\
\hline Abe et al. [16] & PC & G & 1 & 120 & 60 & - & 5 & $0.5 \mathrm{~mL} / \mathrm{kg}$ & 40 & Deconvolution \\
\hline Bize et al. [17] & $\mathrm{AP}$ & $\mathrm{P}$ & 16 & 90 & 100 & 40 & 5 & $40 \mathrm{~mL}$ & 40 & Maximum slope \\
\hline Tsuji et al. [18] & $\mathrm{AP}$ & $\mathrm{T}$ & $16 / 64$ & 120 & $30-50$ & $30-48$ & 4 & $40 \mathrm{~mL}$ & $33-48$ & Deconvolution \\
\hline Tsuji et al. [27] & $\mathrm{AP} / \mathrm{NET}$ & $\mathrm{T}$ & 64 & 80 & 40 & 106 & 4 & $40 \mathrm{~mL}$ & 54 & Deconvolution \\
\hline $\begin{array}{l}\text { Sheiman and } \\
\text { Stick [28] }\end{array}$ & Normal & G & 64 & 100 & 80 & 30 & 4 & $40 \mathrm{~mL}$ & 90 & Compartment \\
\hline $\begin{array}{l}\text { d'Assignies et al. } \\
{[20]}\end{array}$ & NET & G & 64 & 100 & 100 & 70 & 4 & $40 \mathrm{~mL}$ & 150 & Compartment \\
\hline Park et al. [21] & $\mathrm{PC}$ & S & 64 & 100 & 100 & 30 & 5 & $50 \mathrm{~mL}$ & 30 & Patlak plot \\
\hline $\begin{array}{l}\text { Kandel et al. } \\
{[19]}\end{array}$ & PC & $\mathrm{T}$ & 320 & 100 & 45 & 19 & 8 & $60 \mathrm{~mL}$ & 80 & Maximum slope \\
\hline
\end{tabular}

PC: pancreatic cancer; AP: acute pancreatitis; NET: neuroendocrine tumor; S: Siemens; G: GE Health care; P: Philipse; T: Toshiba.

of pancreatic blood flow decreased by more than 19.3\% of surrounding pancreatic parenchyma. The area of perfusion defect was commonly diagnosed by using pancreatic blood flow. The perfusion defects detected by perfusion CT reflected ischemia which was produced by vasospasms of the intrapancreatic arteries $[35,36]$.

\section{Perfusion CT for Evaluating Systemic Blood Flow}

Perfusion CT could be a useful tool to evaluate abnormal systemic circulation in early stage of SAP. Recent study by Whitcomb et al. showed that elevated serum angiopoietin-2 (Ang-2) on admission is predictive of persistent organ failure in patients with sap [37]. Ang-2 is produced by damaged vessels and increases vascular permeability [38]. In our study, elevated serum Ang-2 is related with hyperdynamic state of systemic circulation [22]. In this study, perfusion CT parameter $(\tau)$ was calculated using single-compartment model $[28,39] . \tau$ is a measure of the mean transit time of contrast material from upper abdominal aorta to pancreas; thus, this could be considered a surrogate of systemic circulation with a lower value indicating hyperdynamic state of systemic circulation [28]. In the result, significant correlation was found between $\tau$ and serum Ang-2 levels $(P<0.05)$; higher serum Ang-2 levels were associated with lower $\tau$ values (hyperdynamic state of systemic circulation).

Hepatic circulation abnormality has been reported in patients with SAP using Perfusion CT [40]. They reported that hepatic arterial perfusion is increased in the early stage of SAP as measured on dual-input maximum slope method.

\section{Clinical Utility of Pancreatic Perfusion CT}

Early diagnosis of pancreatic necrosis is very important in the treatment of patients with SAP. Current methods to predict early pancreatic necrosis or SAP is not satisfactory [11-13]. Perfusion CT is a promising technique that allows accurate diagnosis of pancreatic necrosis. Early diagnosis allows prompt clinical decision such as transferring patients to ICU or institution of aggressive treatment such as anticoagulation therapy [41], continuous regional arterial infusion of antiprothrombin agent $[8,9]$, early fluid resuscitation [10], and molecular targeted therapy $[42,43]$.

\section{Conclusion}

Perfusion CT is a promising technique for diagnosis of local and systemic complications of SAP at an early stage.

\section{Conflict of Interests}

The authors declare no conflict of interests.

\section{References}

[1] M. Sekimoto, T. Takada, Y. Kawarada et al., "JPN Guidelines for the management of acute pancreatitis: epidemiology, etiology, natural history, and outcome predictors in acute pancreatitis," Journal of Hepato-Biliary-Pancreatic Surgery, vol. 13, no. 1, pp. 10-24, 2006.

[2] E. L. Bradley, "A clinically based classification system for acute pancreatitis," Annales de Chirurgie, vol. 47, no. 6, pp. 537-541, 1993. 
[3] P. A. Banks, M. L. Freeman, R. Fass et al., "Practice guidelines in acute pancreatitis," American Journal of Gastroenterology, vol. 101, no. 10, pp. 2379-2400, 2006.

[4] S. Tenner, G. Sica, M. Hughes et al., "Relationship of necrosis to organ failure in severe acute pancreatitis," Gastroenterology, vol. 113, no. 3, pp. 899-903, 1997.

[5] C. D. Johnson and M. Abu-Hilal, "Persistent organ failure during the first week as a marker of fatal outcome in acute pancreatitis," Gut, vol. 53, no. 9, pp. 1340-1344, 2004.

[6] N. Takahashi, G. I. Papachristou, G. D. Schmit et al., "CT findings of walled-off pancreatic necrosis (WOPN): differentiation from pseudocyst and prediction of outcome after endoscopic therapy," European Radiology, vol. 18, no. 11, pp. 2522-2529, 2008.

[7] M. S. Petrov, S. Shanbhag, M. Chakraborty, A. R. J. Phillips, and J. A. Windsor, "Organ failure and infection of pancreatic necrosis as determinants of mortality in patients with acute pancreatitis," Gastroenterology, vol. 139, no. 3, pp. 813-820, 2010.

[8] K. Takeda, S. Matsuno, M. Sunamura, and Y. Kakugawa, "Continuous regional arterial infusion of protease inhibitor and antibiotics in acute necrotizing pancreatitis," American Journal of Surgery, vol. 171, no. 4, pp. 394-398, 1996.

[9] M. Piaścik, G. Rydzewska, J. Milewski et al., "The results of severe acute pancreatitis treatment with continuous regional arterial infusion of protease inhibitor and antibiotic: a randomized controlled study," Pancreas, vol. 39, no. 6, pp. 863 $867,2010$.

[10] M. G. Warndorf, J. T. Kurtzman, M. J. Bartel et al., "Early fluid resuscitation reduces morbidity among patients with acute pancreatitis," Clinical Gastroenterology and Hepatology, vol. 9, no. 8, pp. 705-709, 2011.

[11] C. D. Johnson, "UK guidelines for the management of acute pancreatitis," Gut, vol. 54, no. 3, pp. iiil-iii9, 2005.

[12] T. L. Bollen, H. C. Van Santvoort, M. G. Besselink et al., "The Atlanta Classification of acute pancreatitis revisited," British Journal of Surgery, vol. 95, no. 1, pp. 6-21, 2008.

[13] S. Chauhan and C. E. Forsmark, "The difficulty in predicting outcome in acute pancreatitis," American Journal of Gastroenterology, vol. 105, no. 2, pp. 443-445, 2010.

[14] K. A. Miles and M. R. Griffiths, "Perfusion CT: a worthwhile enhancement?" British Journal of Radiology, vol. 76, no. 904, pp. 220-231, 2003.

[15] Y. Tsushima and S. Kusano, "Age-dependent decline in parenchymal perfusion in the normal human pancreas: measurement by dynamic computed tomography," Pancreas, vol. 17, no. 2, pp. 148-152, 1998.

[16] H. Abe, T. Murakami, M. Kubota et al., "Quantitative tissue blood flow evaluation of pancreatic tumor: comparison between xenon CT technique and perfusion CT technique based on deconvolution analysis," Radiation Medicine, vol. 23, no. 5, pp. 364-370, 2005.

[17] P. E. Bize, A. Platon, C. D. Becker, and P. A. Poletti, "Perfusion measurement in acute pancreatitis using dynamic perfusion MDCT," American Journal of Roentgenology, vol. 186, no. 1, pp. 114-118, 2006.

[18] Y. Tsuji, H. Yamamoto, S. Yazumi et al., "Perfusion computerized tomography can predict pancreatic necrosis in early stages of severe acute pancreatitis," Clinical Gastroenterology and Hepatology, vol. 5, no. 12, pp. 1484-1492, 2007.

[19] S. Kandel, C. Kloeters, H. Meyer, P. Hein, A. Hilbig, and P. Rogalla, "Whole-organ perfusion of the pancreas using dynamic volume CT in patients with primary pancreas carcinoma: acquisition technique, post-processing and initial results," European Radiology, vol. 19, no. 11, pp. 2641-2646, 2009.

[20] G. d'Assignies, A. Couvelard, S. Bahrami et al., "Pancreatic endocrine tumors: tumor blood flow assessed with perfusion CT reflects angiogenesis and correlates with prognostic factors," Radiology, vol. 250, no. 2, pp. 407-416, 2009.

[21] M. S. Park, E. Klotz, M. J. Kim et al., "Perfusion CT: noninvasive surrogate marker for stratification of pancreatic cancer response to concurrent chemo-And radiation therapy," Radiology, vol. 250, no. 1, pp. 110-117, 2009.

[22] T. Watanabe, Y. Tsuji, Y. Kodama, H. Isoda, H. Yamamoto, and T. Chiba, "Relationship between serum angiopoietin-2 level and perfusion CT parameters in severe acute pancreatitis," American Journal of Gastroenterology, vol. 106, no. 10, pp. 1859-1861, 2011.

[23] Y. Tsushima, M. Miyazaki, A. Taketomi-Takahashi, and K. Endo, "Feasibility of measuring human pancreatic perfusion in vivo using imaging techniques," Pancreas, vol. 40, no. 5, pp. 747-752, 2011.

[24] D. V. Sahani, N. S. Holalkere, A. Kambadakone, K. Matthes, M. Mino-Kenudson, and W. R. Brugge, "Role of computed tomography perfusion in the evaluation of pancreatic necrosis and pancreatitis after endoscopic ultrasound-guided ablation of the pancreas in a porcine model," Pancreas, vol. 38, no. 7, pp. 775-781, 2009.

[25] K. A. Miles, "Perfusion CT for the assessment of tumour vascularity: which protocol?" British Journal of Radiology, vol. 76, no. 1, pp. S36-S42, 2003.

[26] M. Kishimoto, Y. Tsuji, N. Katabami et al., "Measurement of canine pancreatic perfusion using dynamic computed tomography: influence of input-output vessels on deconvolution and maximum slope methods," European Journal of Radiology, vol. 77, pp. 175-181, 2009.

[27] Y. Tsuji, K. Koizumi, H. Isoda et al., "The radiological exposure of pancreatic perfusion computed tomography," Pancreas, vol. 39, no. 4, p. 541, 2010.

[28] R. G. Sheiman and A. Sitek, "Feasibility of measurement of pancreatic perfusion parameters with single-compartment kinetic model applied to dynamic contrast-enhanced CT images," Radiology, vol. 249, no. 3, pp. 878-882, 2008.

[29] V. Goh, M. Dattani, J. Farwell et al., "Radiation dose from volumetric helical perfusion CT of the thorax, abdomen or pelvis," European Radiology, vol. 21, pp. 974-981, 2011.

[30] A. R. Kambadakone, A. Sharma, O. A. Catalano, P. F. Hahn, and D. V. Sahani, "Protocol modifications for CT perfusion (CTp) examinations of abdomen-pelvic tumors: impact on radiation dose and data processing time," European Radiology, vol. 21, no. 6, pp. 1293-1300, 2011.

[31] P. C. Shrimpton, M. C. Hillier, M. A. Lewis, and M. Dunn, "National survey of doses from CT in the UK: 2003," British Journal of Radiology, vol. 79, no. 948, pp. 968-980, 2006.

[32] L. Yu, H. Li, J. G. Fletcher, and C. H. McCollough, "Automatic selection of tube potential for radiation dose reduction in CT: a general strategy," Medical Physics, vol. 37, no. 1, pp. 234-243, 2010.

[33] T. K. Leung, C. M. Lee, S. Y. Lin et al., "Balthazar computed tomography severity index is superior to Ranson criteria and APACHE II scoring system in predicting acute pancreatitis outcome," World Journal of Gastroenterology, vol. 11, no. 38, pp. 6049-6052, 2005.

[34] J. P. Neoptolemos, E. A. Kemppainen, J. M. Mayer et al., "Early prediction of severity in acute pancreatitis by urinary trypsinogen activation peptide: a multicentre study," The Lancet, vol. 355, no. 9219, pp. 1955-1960, 2000. 
[35] K. Takeda, Y. Mikami, S. Fukuyama et al., "Pancreatic ischemia associated with vasospasm in the early phase of human acute necrotizing pancreatitis," Pancreas, vol. 30, no. 1, pp. 40-49, 2005.

[36] Y. Tsuji, K. Hamaguchi, Y. Watanabe et al., "Perfusion CT is superior to angiography in predicting pancreatic necrosis in patients with severe acute pancreatitis," Journal of Gastroenterology, vol. 45, pp. 1155-1162, 2010.

[37] D. C. Whitcomb, V. Muddana, C. J. Langmead et al., "Angiopoietin-2, a regulator of vascular permeability in inflammation, is associated with persistent organ failure in patients with acute pancreatitis from the United States and Germany," American Journal of Gastroenterology, vol. 105, no. 10, pp. 2287-2292, 2010.

[38] U. Fiedler, Y. Reiss, M. Scharpfenecker et al., "Angiopoietin-2 sensitizes endothelial cells to TNF- $\alpha$ and has a crucial role in the induction of inflammation," Nature Medicine, vol. 12, no. 2, pp. 235-239, 2006.

[39] A. Sitek and R. G. Sheiman, "Small-bowel perfusion measurement: feasibility with single-compartment kinetic model applied to dynamic contrast-enhanced CT," Radiology, vol. 237, no. 2, pp. 670-674, 2005.

[40] S. Koyasu, H. Isoda, Y. Tsuji et al., "Hepatic arterial perfusion increases in the early stage of severe acute pancreatitis patients: evaluation by perfusion computed tomography," European Journal of Radiology, vol. 81, no. 1, pp. 43-46, 2012.

[41] T. Hackert, J. Werner, W. Uhl, M. M. Gebhard, M. W. Büchler, and J. Schmidt, "Reduction of ischemia/reperfusion injury by antithrombin III after experimental pancreas transplantation," American Journal of Surgery, vol. 189, no. 1, pp. 92-97, 2005.

[42] X. Wang, W. Li, C. Niu, L. Pan, N. Li, and J. Li, "Thymosin alpha 1 is associated with improved cellular immunity and reduced infection rate in severe acute pancreatitis patients in a double-blind randomized control study," Inflammation, vol. 34, pp. 198-202, 2011.

[43] M. Tidswell, W. Tillis, S. P. Larosa et al., "Phase 2 trial of eritoran tetrasodium (E5564), a Toll-like receptor 4 antagonist, in patients with severe sepsis," Critical Care Medicine, vol. 38, no. 1 , pp. 72-83, 2010. 


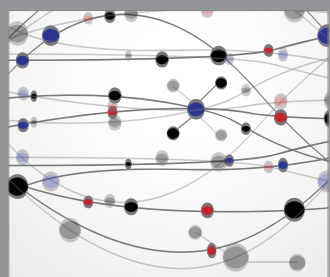

The Scientific World Journal
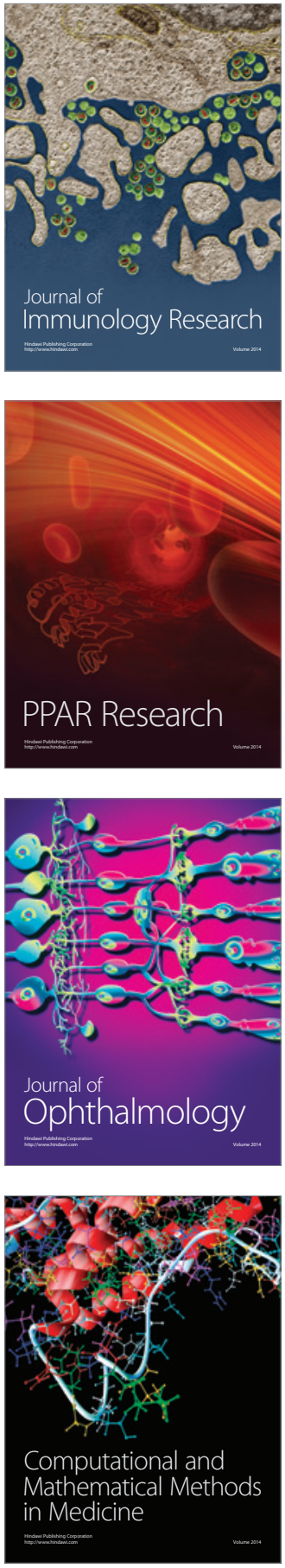

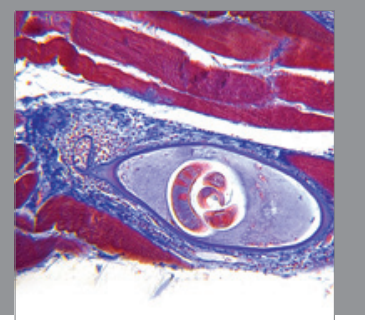

Gastroenterology

Research and Practice
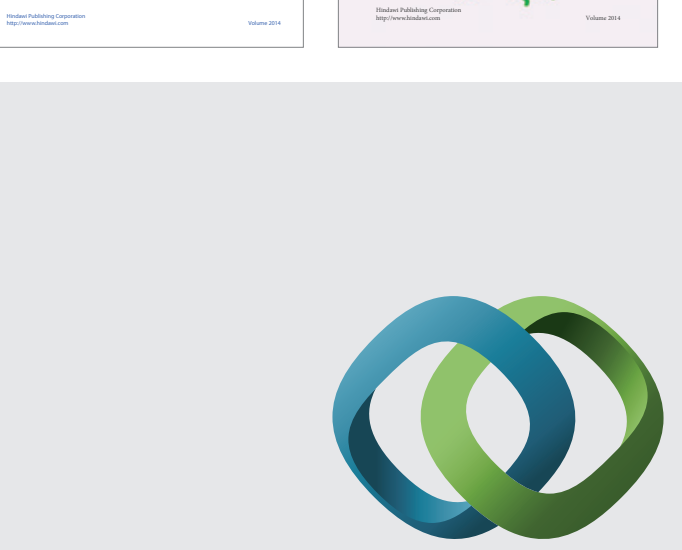

\section{Hindawi}

Submit your manuscripts at

http://www.hindawi.com
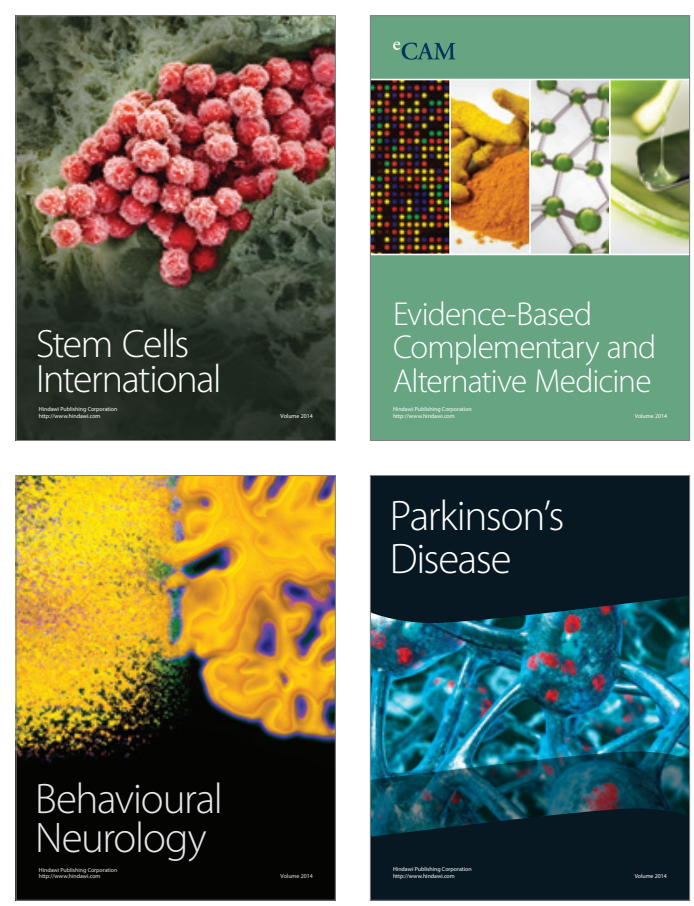

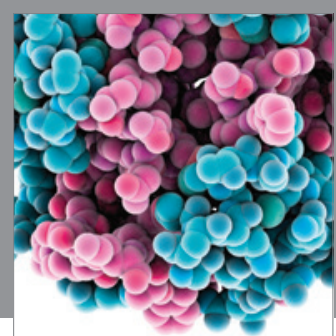

Journal of
Diabetes Research

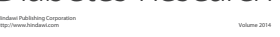

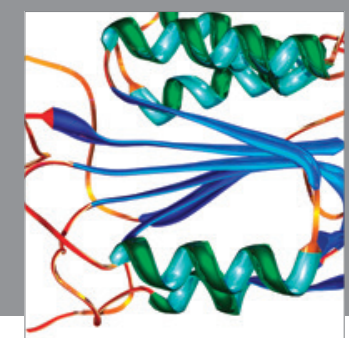

Disease Markers
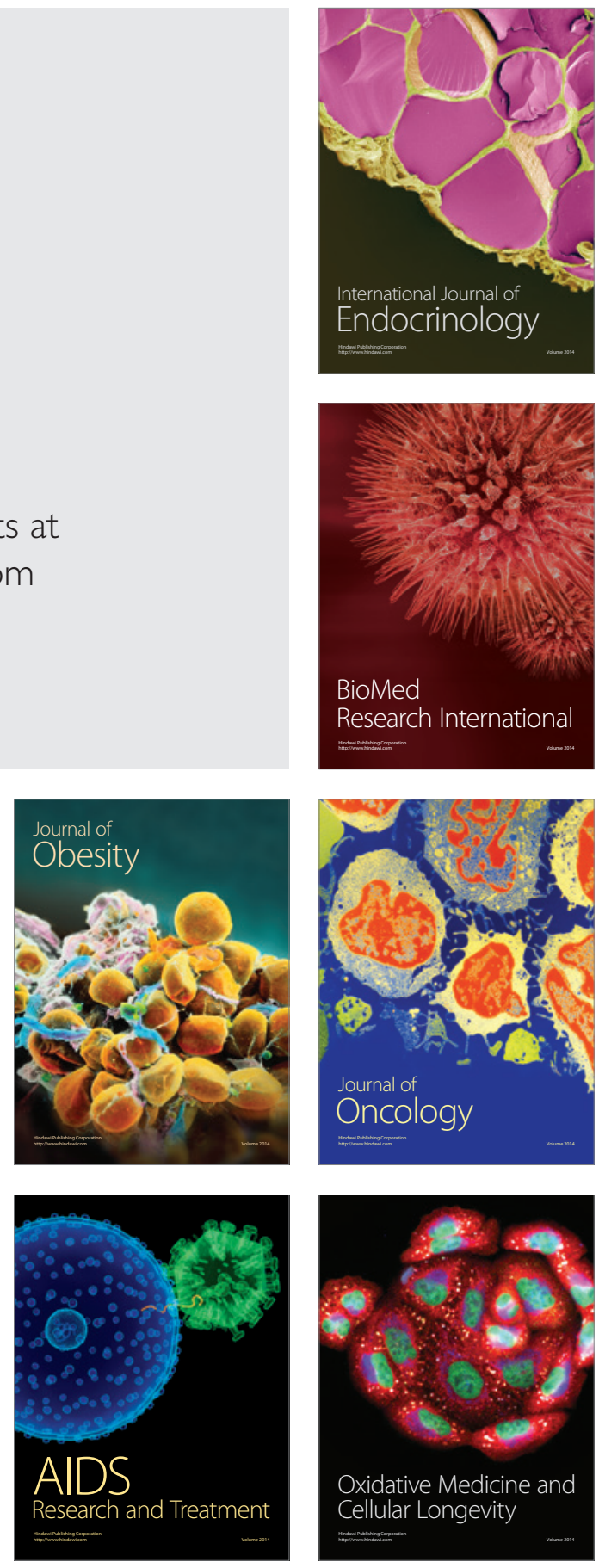